\title{
Perfil celular e microbiológico do leite de ovelhas Santa Inês no período lactante e pós-desmame ${ }^{1}$
}

\author{
Maiara G. Blagitz ${ }^{*}$, Camila F. Batista ${ }^{2}$, Fernando N. Souza², Nilson R. \\ Benites $^{3}$, Priscila A. Melville ${ }^{3}$, Claúdia R. Stricagnolo², Mariana Ricciardi², \\ Viviani Gomes ${ }^{2}$, Milton R. Azedo², Bárbara G.S. Sanches ${ }^{2}$ e Alice M.M.P. \\ Della Libera ${ }^{2}$
}

\begin{abstract}
Blagitz M.G., Batista C.F., Souza F.N., Benites N.R., Melville P.A., Stricagnolo C.R., Ricciardi M., Gomes V., Azedo M.R., Sanches B.G.S. \& Della Libera A.M.M.P. 2008. [Cellular and microbiological profile of Santa Ines ewes in the lactation and the post-weaning period.] Perfil celular e microbiológico de ovelhas Santa Inês no período lactante e pós-desmame. Pesquisa Veterinária Brasileira 28(9):417422. Departamento de Clínica Médica, Faculdade de Medicina Veterinária e Zootecnia, Universidade de São Paulo, Av. Prof. Dr. Orlando Marques de Paiva 87, Cidade Universitária, São Paulo, SP 05508-270, Brazil. E-mail: magblagitz@usp.br

The aim was to evaluate and compare the microbiological and cellular profile of the milk of Santa Ines ewes during the lactation period and the active involution. Milk samples were analyzed from 12 ewes during these distinct periods. Clinical examination of the mammary gland, somatic cell count (SCC), California Mastitis Test (CMT), bacteriologic screening and sensibility of the pathogens in vitro were performed. Most alterations were observed in the active involution period. SCC and CMT were higher in this same period. Besides this, a high persistency of infection occurred. The active involution period did not show high susceptibility. Coagulase-negative staphylococci were the only isolated bacteria. A high antimicrobial sensibility of these pathogens was also encountered.
\end{abstract}

INDEX TERMS: Mastitis, ewes, involution, lactation, staphylococci.

RESUMO.- O objetivo deste trabalho foi avaliar o perfil microbiológico e celular do leite no período lactante e de involução ativa de ovelhas da raça Santa Inês. Foram avaliadas amostras lácteas de 12 ovelhas durante estes distintos períodos. Realizou-se o exame físico da mama, sendo as amostras lácteas submetidas à contagem de células somáticas (CCS), ao California Mastitis Test (CMT), ao exame microbiológico e aos testes de sensibi-

\footnotetext{
${ }^{1}$ Recebido em 29 de agosto de 2007.

Aceito para publicação em 12 de setembro de 2008.

Parte integrante da Dissertação da primeira autora, apresentada ao Programa de Pós-Graduação em Clínica Médica da Faculdade de Medicina Veterinária e Zootecnia, Universidade de São Paulo (USP).

${ }^{2}$ Departamento de Clínica Médica, Faculdade de Medicina Veterinária e Zootecnia, USP, Av. Prof. Dr. Orlando Marques de Paiva 87, Cidade Universitária, São Paulo, SP 05508-270, Brasil. *Autor para correspondência: magblagitz@usp.br

${ }^{3}$ Departamento de Medicina Veterinária Preventiva e Saúde Animal, Faculdade de Medicina Veterinária e Zootecnia, USP, São Paulo, SP.
}

lidade in vitro dos patógenos encontrados. Foram observados maiores escores do exame físico, CCS, CMT durante o período de involução ativa, além de uma alta persistência da infecção durante estes períodos. O período de involução ativa não se mostrou como um momento de alta susceptilidade. Os estafilococos coagulase negativa representaram o único gênero isolado das glândulas infectadas. Uma alta sensibilidade dos agentes etiológicos envolvidos frente aos diferentes antimicrobianos in vitro foi também observada.

TERMOS DE INDEXAÇÃO: Mastite, ovelhas, involução, lactação, estafilococos.

\section{INTRODUÇÃO}

A mamite é um processo inflamatório mamário usualmente causado por bactérias. Os estudos relacionados a esta doença se iniciaram há pouco mais de um século, tendo uma vasta literatura acumulada sobre o assunto, porém grande parte destes estudos se concentram na espécie 
bovina. Deve-se salientar ainda que os ovinos apresentam importância econômica em vários países e o seu número tem aumentado (Burriel 1997).

Esta doença é a principal razão para o descarte de ovelhas, tendo as formas clínicas inaparentes, o maior efeito na redução da produção e na qualidade do leite, levando a prejuízos maiores que aqueles observados em bovinos leiteiros (Watson \& Buswell 1984, Burriel 1997, Leitner et al. 2001, Gonzalo et al. 2002). Esta classificação da enfermidade é considerada a mais prevalente entre estes animais (Contreras et al. 2007).

A mastite também está associada com a performance dos cordeiros (Burris \& Baugus 1955, Torre-Hernandez \& Hohenboken 1979, Fthenakis \& Jones 1990) descarte de ovelhas, mortes (Watson \& Buswell 1984), custos com tratamentos (Keisler et al. 1992), gastos adicionais com mão-de-obra (Larsgrad \& Vaabenoe 1993) e alteração na composição do leite (Green 1984, Hueston et al. 1986b).

Este processo inflamatório pode ser diagnosticado pela inspeção e palpação, e ainda por exames complementares que avaliem a celularidade do leite $\mathrm{e} o$ isolamento de agentes patogênicos (Keisler et al. 1992).

O desmame acarreta mudanças no tecido mamário e início do processo denominado involução mamária (Hurley 1989). O período seco ainda é dividido em três fases: período de involução ativa, período de involução gradual e o período de colostrogênese e lactogênese (Hurley 1999). As alterações na composição da secreção mamária que ocorrem durante os estágios iniciais da involução indicam rápidas mudanças estruturais na glândula e nos mecanismos envolvidos na secreção e síntese láctea (Holst et al. 1987, Oliver \& Sordillo 1989, Rejman et al. 1989, Hurley 1989). A involução ativa inicia-se logo após o desmame, resultando em estase láctea com concomitante aumento de volume, declinando este rapidamente nas próximas semanas (Smith \& Todhunter 1982, Hurley 1999). Estas mudanças influenciam a resposta imune local (Lee \& Outteridge 1981, Tatarczuch et al. 1997, 2000), como a redução da função fagocítica neste período (Tatarczuch et al. 2002). No entanto, a variação na susceptibilidade também pode ser influenciada pela habilidade da secreção mamária de inibir o crescimento bacteriano (Dutt et al. 1986).

Com proposta para reduzir a persistência das infecções pré-existentes e prevenir novas infecções tem sido sugerido o tratamento no período seco (Hueston et al. 1989, Chaffer et al. 2003, Gonzalo et al. 2004), sendo que muitas vezes o sucesso de terapia está relacionado com os patógenos encontrados (Hueston et al. 1989, Bergonier et al. 2003). Com o intuito de avaliar a eficácia dos tratamentos, a utilização de antibiogramas na seleção de drogas empregadas na terapia tem indicação (Craven 1987, Erskine et al. 2004).

O presente estudo teve como objetivo avaliar o perfil celular e microbiólogico do leite de ovelhas da raça Santa Inês, com o intuito de observar a susceptibilidade do período de involução ativa da glândula mamária e presença de resistência antimicrobiana in vitro.

\section{MATERIAL E MÉTODOS}

Foram selecionadas de forma casualizada 12 ovelhas da raça Santa Inês, as quais tiveram seus úberes examinados e amostras de leite coletadas de forma pareada em dois momentos. $O$ primeiro momento foi no período lactante com seu cordeiro em amamentação (12 dias anterior ao desmame) e o segundo foi após o desmame abrupto dos cordeiros (7 dias após o desmame).

Conforme o exame físico (Baumgartner 2005), os mesmos foram classificados em escores onde 1 correspondeu às mamas com úberes e tetos normais na inspeção e palpação, 2 para mamas com úberes e/ou tetos alterados à inspeção e normais à palpação, 3 para mamas com úberes e/ou tetos normais à inspeção e alterados à palpação, e 4 para úberes e/ou tetos alterados à inspeção e palpação.

Após as amostras terem sido submetidas a prova do fundo escuro, estas foram coletadas assepticamente e foram submetidas ao exame microbiológico, CMT (Schalm \& Noorlander 1957) e contagem microscópica direta de células somáticas.

No exame microbiológico as amostras de leite foram semeadas em placa de Petri contendo ágar-sangue de carneiro $(5 \%)$ e incubadas a $37^{\circ} \mathrm{C}$ por 24 e 48 horas, para posterior identificação dos microrganismos através de provas bioquímicas descritas por Lennette et al. (1985), seguida da classificação conforme critérios estabelecidos por Krieg \& Holt (1994). A contagem de células somáticas foi realizada pela microscopia através de esfregaços corados com Hematoxilina-Eosina (Prescott \& Breed 1910, Benites et al. 2001), onde foram contados 100 campos e o resultado submetido ao fator microscópico de 3571.

Foram também realizados testes de sensibilidade in vitro dos microrganismos isolados do leite frente a amoxicilina, ampicilina, cefalotina, gentamicina, estreptomicina, neomicina, oxacilina, penicilina, sulfonamida e tetraciclina, sendo estes organismos classificados em sensíveis ou resistentes (Bauer et al. 1966).

O teste estatístico de Friedman foi utilizado para a análise das variáveis testadas.

\section{RESULTADOS}

Durante a inspeção, 11 mamas e 17 tetos que apresentaram normais durante a lactação se mantiveram normais após a secagem. Das 13 mamas e 5 tetos que apresentaram normais durante a lactação apresentaram alterados após a secagem. Todas as mamas apresentaram aumentadas de volume e 3 tetos apresentaram aumentados de volume e 2 apresentaram diminuídos de volume e dos tetos que estavam normais, apenas 2 apresentaram aumentados de volume após a secagem.

Durante a palpação, 6 mamas e 21 tetos que apresentaram normais durante a lactação se mantiveram normais após a secagem. Das 12 mamas e dos 2 tetos que apresentaram normais durante a lactação apresentaram alterados após a secagem. Uma mama apresentou consistência normal mas com a presença de nódulos pequenos e 11 apresentaram consistência firme, os dois tetos apresentaram telite. Apenas 2 mamas e 1 teto apresentaram alterados durante a lactação e normais após a secagem. As duas mamas apresentaram consistência normal mas com nódulos endurecidos enquanto que o teto apresen- 
Quadro 1. Exame físico e perfil celular de ovelhas no final da lactação e no início do período seco

\begin{tabular}{lcccccc}
\hline \multicolumn{1}{c}{ Ovelhas } & $\mathrm{N}$ & EEF & CCS & CMT & $\% \mathrm{PMN}$ & $\% \mathrm{MN}$ \\
\hline Lactantes negativas & 12 & 1 & 35.710 & 0 & 12,78 & 87,22 \\
Lactantes positivas & 12 & 2 & 857.040 & 1 & 17,28 & 82,72 \\
Secas negativas & 13 & 3 & 171.408 & 1 & 14,85 & 85,15 \\
Secas positivas & 11 & 4 & 1.978 .334 & 2 & 25 & 75,00 \\
Valor de significância & & 0,005 & 0,06 & 0,17 & 0,07 & 0,012
\end{tabular}

tou telite. Das 4 mamas que apresentara alteradas na lactação persistiram alteradas após a secagem, uma delas apresentava consistência macia com nódulos endurecidos e na secagem os nódulos tornaram-se pequenos e 3 mamas que apresentaram consistência macia com nódulos endurecidos apresentaram consistência firme.

Os escores do exame físico (EEF), assim como os valores da mediana da contagem microscópica de células somáticas, da porcentagem de mononucleares e polimorfonucleares e do CMT encontram-se no Quadro 1.

Nas provas microbiológicas foram observadas durante a lactação que $50 \%$ das amostras foram positivas e destas foram isoladas apenas Staphylococcus spp. As 12 amostras positivas foram resistentes a sulfonamida e/ ou tetraciclina na fase lactante. Durante a fase pós-desmame, $46 \%$ das amostras foram positivas e destas foram isoladas apenas Staphylococcus spp. As 11 amostras positivas foram resistentes a sulfonamida e penicilina, sendo que uma delas apresentou resistência ainda para amoxicilina, ampicilina, estreptomicina e oxaciclina.

Encontrou-se também uma persistência do quadro infeccioso de $87,5 \%$, sendo que 2 tetos infectados durante a fase lactante obtiveram cura espontânea na fase pósdesmame e apenas um teto se infectou durante estes períodos, considerando-se o exame microbiológico como o teste de referência para o diagnóstico de mamite infecciosa.

\section{DISCUSSÃO}

A fisiologia do período seco diverge bastante com a do período lactacional (Hurley 1999), sendo que após a secagem o úbere continua a sintetizar e secretar leite ocorrendo acúmulo máximo no segundo e terceiro dia após a secagem (Nickerson 1989). Todavia, durante o início deste período há um acúmulo de fluidos e concomitante aumento de componentes antibacterianos, leucócitos, imunoglobulinas e lactoferrina na tentativa de reduzir o volume de leite produzido (Smith \& Todhunter 1982). A composição da secreção mamária é importante em termos da presença de fatores que conferem resistência a glândula mamária durante este período. Mudanças na composição podem refletir mudanças em funções celulares que ocasionam implicações na resistência da glândula mamária (Hurley 1989, Tatarczuch et al.1997, 2000, 2002).

As diferenças encontradas durante os distintos períodos, sugerem que a análise dos diferentes aspectos da glândula mamária ovina devem ser cuidadosamente avaliadas, por exemplo, pode-se esperar alterações no exa- me físico, onde se observou um aumento de dois escores desta avaliação independente do estado infeccioso do animal.

Congruente a isto, o aumento na CCS destes animais no período pós-desmame, pode ser explicado pelo aumento fisiológico de leucócitos neste período com concomitante decréscimo do volume (Lee \& Lascelles 1969, Smith \& Todhunter 1982). Este aumento na CCS poderia ainda explicar o aumento de um escore no CMT, independente do quadro infeccioso do animal, já que este está altamente correlacionado com a CCS (Mcdougall et al. 2001). Este aumento está intimamente correlacionado com o aumento da defesa imunitária próximo à secagem, quando a glândula mamária necessita se recuperar das infecções existentes e evitar novas infecções (Cuccuru et al. 1997).

Observou-se também um aumento na porcentagem de polimorfonucleares nas mamas infectadas nos dois períodos, o que explicaria o aumento na CCS na infecção mamária, onde há uma alta correlação entre PMN e aumento na CCS (Bergonier et al. 2003). Os neutrófilos polimorfonucleares (PMN) são a primeira linha de defesa celular contra a invasão de agentes patogênicos (Paape et al. 2002), acumulando rapidamente no sítio da infecção (Capuco et al. 1986). Contudo, observou-se apenas um aumento discreto na porcentagem de polimorfonucleares no período involutivo nas ovelhas sadias ao confrontarmos com os animais sadios lactantes, já que se espera um aumento fisiológico nos leucócitos $\mathrm{PMN}$ durante o período involutivo e colostral (Bergonier et al. 2003).

Foi encontrado também uma persistência do quadro infeccioso nestes animais. Deste modo, quase não houve mudanças no quadro infeccioso da glândula mamária ovina. Concordante a isto, Watkins et al. (1991), Leitner et al. (2001) e Al-Majali \& Jawabreh (2003) reportaram que a prevalência da infecção permaneceu constante durante toda a lactação, apesar de Hueston et al.(1986a) e Al-Samarrae et al.(1985) terem relatado que a prevalência aumenta. Isto no entanto, pode ser devido aos diferentes meios diagnósticos utilizados, o que dificulta comparações entre os estudos. Deve-se lembrar também que vários fatores como manejo, raça, clima e nutrição podem influenciar na incidência das infecções mamárias ovinas (Fthenakis \& Jones 1990).

A presença de infecção intramamária durante o período lactacional pode ser variável de acordo com o patógeno envolvido, mas é geralmente alta ao considerar que os estafilococos representam o grupo mais frequentemente isolado (Saratsis et al. 1999, Leitner et al. 2001, Bergonier et al. 2003). As infecções intramamárias) (IIM) não são geralmente detectadas e a sua eliminação não ocorre durante a lactação (Bergonier et al. 2003). A persistência das IIM, durante o período seco é importante ao considerarmos estratégias de tratamento e uma taxa de cura espontânea de 35-67\% (Hueston et al. 1989, Bergonier et al. 2003).

Todas as amostras positivas nas provas bacteriológi- 
cas isolaram Staphylococcus spp. coagulase-negativa $(\mathrm{ECN})$, sendo que estas correspondem à principal causa dos processos infecciosos em ovelhas (Hueston et al. 1986a, 1989, Bor et al. 1989, Watkins et al. 1991, Kirk et al. 1996, Menzies \& Ramanoon 2001, Ariznabarreta et al. 2002, Bergonier et al. 2003, Contreras et al. 2007). As infecções por estes patógenos geralmente persistem do período seco até a próxima lactação (Poutrel 1984, Nickerson 1987). Staphylococcus aureus, Streptococcus spp., Manhemia haemolytica, Pseudomonas aeroginosa, Enterobacteriaceae spp., Corynebacteria spp., Micrococci spp. e alguns fungos, são também identificados como patógenos causadores de mastite crônica nestes animais (Al-Samarrae et al. 1985, Watkins et al. 1991, Della Libera et al. 2007).

Ao avaliar a susceptibilidade microbiana frente a diferentes antimicrobianos, observou-se uma boa sensibilidade destes microrganismos. Esta sensibilidade da microbiota isolada pode indicar uma boa resposta ao tratamento destes animais (Craven 1987, Erskine et al. 2004). Uma boa sensibilidade bacteriana também foi encontrada por Gutierrez et al.(1990) e Fthenakis (1998). Assim, Hueston et.al. (1986a) atribui baixa virulência a cepas de estafilococos coagulase-negativa, relatando a possibilidade da resolução do processo infeccioso sem tratamento, além do sucesso encontrado na terapia antimicrobiana intramamária no final da lactação, possivelmente relacionada ao impacto destes estafilococos. Contudo, Saratsis et al. (1998) reportou um aumento na resistência antimicrobiana destes agentes etiológicos. Costa et al. (2000) também relatou uma alta resistência destes agentes frente aos antimicrobianos testados em amostras provenientes de glândulas mamárias bovinas.

Melchior et al. (2006) considerou a susceptibilidade do agente ao antimicrobiano é um preditor pobre para cura bacteriológica. Além disso, os dados de ampicilina, por exemplo, utilizados na determinação do limiar de susceptibilidade/resistência bacterianas são provenientes de enfermidades humanas (Erskine et al. 2004). Existem ainda situações como a aparente sensibilidade de $S$. aureus que não necessariamente indica que haverá sucesso na terapia (Craven 1987).

Não houve indícios para afirmar que o período de involução ativa apresenta como período crítico de susceptibilidade a infecção mamária em ovinos Santa Inês, corroborando com Bergonier et al. (2003) que condicionou a alta incidência na secagem e na proximidade ao parto a casos específicos e raros (Pseudomonas spp. e micocoses), ou a práticas higiênicas precárias, sendo considerado o terço inicial da lactação como o período de maior susceptibilidade (Kirk et al 1996, Leitner et al 2001, Bergonier et.al 2003), apesar de Saratsis et al.(1998) afirmar que o período involutivo apresenta uma alta susceptibilidade. Congruente a isto, Kirk et al.(1996) encontrou uma alta taxa de cura espontânea após o desmame. Estudos analisando todo o ciclo lactacional são necessários para se determinar os períodos de maior susceptibidade à mastite. Além disso, a alta sensibilidade bacteriana frente aos antimicrobianos in vitro encontrada deve ser avaliada em estudos que verifiquem a eficácia de tratamentos da mastite ovina frente a estes microbianos.

É também relatado que a secreção mamária obtida durante o período de involução ativa é um meio pobre para o crescimento de espécies de Staphylococcus spp. (Oliver \& Sordillo 1989), sendo relatado também uma alta taxa de cura por estafilococos coagulase negativo durante o período não lactante (Harmon et al. 1986), sugerindo que a habilidade da secreção mamária de inibir o crescimento bacteriano pode estar correlacionada com as taxas de novas IIM que ocorrem durante o período seco (Dutt et al. 1986, Harmon et al. 1986). Estes dados são ainda coerentes com Mcdonald \& Anderson (1983) que observaram zero de nove quartos infectados após inoculação de Staphylococcus epidermidis no sétimo dia após a secagem, e 36 de 38 infectadas durante a metade final do período involutivo. Isto indica que outros fatores que conferem proteção podem estar envolvidos e apresentarem uma importante proteção às IIM (Dutt et al. 1986). Isto poderia ser a razão pelo qual este período não se mostrou como um período de alta susceptibilidade.

\section{CONCLUSÕES}

A involução não apresentou-se como um período de alta susceptibilidade a IIM em ovinos Santa Inês.

Observou-se um aumento no recrutamento celular durante este período, o que justificaria a maior CCS e CMT encontradas.

Em relação ao exame físico, as anormalidades devem ser criteriosamente analisadas, já que se encontraram maiores escores ao exame físico no período de involução ativa independente da presença de IIM ao confrontarmos com o período lactacional.

Os estafilococos coagulase-negativa apresentaram uma alta sensibilidade antimicrobiana in vitro o que indica um sucesso na antimicrobianoterapia, sendo estes grandes responsáveis pelas IIM nestes agentes.

Agradecimentos.- À Fundação de Amparo à Pesquisa do Estado de São Paulo (FAPESP) pelo apoio financeiro.

\section{REFERÊNCIAS}

Al-Majali A.M. \& Jawabreh S. 2003. Period prevalence and etiology of subclinical mastitis in Awassi sheep in southern Jordan. Small Ruminant Research 47:243-248.

Al-Samarrae S.E.G., Sharma V.K. \& Yousif A.A. 1985. Mastitis in sheep in Iraq. Vet. Rec. 116:323.

Ariznabarreta A., Gonzalo C. \& San Primitivo F. 2002. Microbiological quality and somatic cell count of ewe milk with special reference to staphylococci. J. Dairy Sci. 85:1370-1375.

Bauer A.W., Kirby W.M., Sherris J.C. \& Turck M. 1966. Antibiotic susceptibility tests by standardized single disk method. Am. J. Clin. Pathol. 45:493-496.

Baumgartner W. 2005. Klinische Propädeutik der inneren Krankheiten und Hautkrankheiten der Haus- und Heimtiere. 6.Aufl. Parey Verlag, Berlin. 382 p. 
Benites N.R., Melville P.A. \& Costa E.O. 2001. Modificação da técnica de contagem de células somáticas de Prescott \& Breed utilizando-se a coloração de Hematoxilina e Eosina. Napgama 4(3):6-9.

Bergonier D., Crémoux R., Rupp R., Lagriffoul G. \& Berthelot X. 2003. Mastitis of dairy small ruminants. Vet. Res. 34:689-716.

Bor A., Winkler M. \& Gootwine E. 1989. Non-clinical intramammary infections in lactating ewes and its association with clinical mastitis. Brit. Vet. J. 145:178-184.

Burriel A.R. 1997. Dynamics of intramammary infection in the sheep caused by coagulase-negative staphylococci and its influence on udder tissue and milk composition. Vet. Rec. 140:419-423.

Burris M.J. \& Baugus C.A. 1955. Milk consumption and growth of suckling lambs. J. Anim. Sci. 14:186-191.

Capuco A.V., Paape M.J. \& Nickerson S.C. 1986. In vitro study of polymorphonuclear leukocyte damage to mammary tissue of lactating cows. Am. J. Vet. Res. 47:663- 668.

Chaffer M., Leitner G., Zamir S., Winkler M., Glickman A., Ziv N. \& Saran A. 2003. Efficacy of the dry-off treatment in sheep. Small Ruminant Research 47:11-16.

Contreras A., Sierra D., Sánchez A., Corrales J.C., Marco J.C., Paape M.J. \& Gonzalo C. 2007. Mastitis in small ruminants. Small Ruminant Research 68:145-153.

Costa E.O., Benites N.R. \& Guerra J.L. \& Melville P.A. 2000. Antimicrobial susceptibility of Staphylococcus spp. isolated from mammary parenchymas slaughtered dairy cows. J. Vet. Med. B 47:99-103.

Craven N. 1987. Efficacy and financial value of antibiotic treatment of bovine clinical mastitis during lactation: A review. Brit. Vet. J. 147:410422.

Cuccuru C., Moroni P., Zecconi A., Casu S., Caria A. \& Contini A. 1997. Milk differential cell counts in relation to total counts in Sardinian ewes. Small Ruminant Research 25:169-173.

Della Libera A.M.M., Blagitz M.G. \& Azedo M.R. 2007. Mastite de pequenos ruminantes. Anais $4^{\circ}$ Encontro de Pesquisadores em Mastites, Botucatu, São Paulo, p.38-44.

Dutt K.W., Eberhart R.J. \& Wilson R.A. 1986. In vitro growth of mastitis pathogens in mammary gland secretions of the dry and peripartum periods. J. Dairy Sci. 69(9):2408-2415.

Erskine R., Cullor J., Schaellibaum M., Yancey B. \& Zecconi A. 2004. Bovine mastitis pathogens and trends in resistence to antimicrobial drugs. Annual Meeting of the National Mastitis Council, p.400-414. Disponível em < http://www.nmconline.org/docs/ResPaper.pdf $>$ Acesso em 15.7.2007.

Fthenakis G.C. \& Jones J.E.T. 1990. The effect of experimental induced clinical mastitis on milk yield of ewes and on the growth of lambs. Brit. Vet. J. 146:43-49.

Fthenakis G.C. 1998. Susceptibility to antibiotics of staphylococcal isolates from cases of ovine or bovine mastitis in Greece. Small Ruminant Research 28:9-13.

Gonzalo C., Ariznabarreta A. \& Carriedo J.A. 2002. Mammary pathogens and their relationship to somatic cell count and milk yield losses in dairy ewes. J. Dairy Sci. 85:1460-1467.

Gonzalo C., Tardáguila J.A., De La Fuente L.F. \& San Primitivo F. 2004. Effect of selective and complete dry therapy on prevalence of intramammary infection and on milk yield in the subsequentlactation in dairy ewes. J. Dairy Res. 71:33-38.

Green T.J. 1984. Use of somatic cell counts for detection of subclinical mastitis in ewes. Vet. Rec. 114:43.

Gutiérrez L.M., Garciá M.L. \& Otero M.C. 1990. Incidence of staphylococci in ovine mastitic milk and antibiotic susceptibility of the strains. Milchwissenschaft 45:778-781.

Harmon R.J., Crist W.L., Hemken R.W. \& Langlois B.E. 1986. Prevalence of minor pathogens after intramammary dry therapy. J. Dairy Sci. 69:843-849.
Holst B.D., Hurley W.L. \& Nelson D.R. 1987. Involution of the bovine mammary gland: Histological and ultrastructural changes. J. Dairy Sci. 70:935-944

Hueston W.D., Hartwing N.R. \& Judy J.K. 1986a. Patterns non-clinical intramammary infection in a ewe floc. J. Am.n Vet. Med. Assoc. 188:170-172.

Hueston W.D., Hartwing N.R. \& Judy J.K. 1986b. Detection of ovine intramammary infection with the California Mastitis Test. J. Am. Vet. Med. Assoc. 188:522-524.

Hueston W.D., Boner G.J. \& Baertsche S.L. 1989. Intramammary antibiotic treatment at the end of lactation for prophylaxis and treatment of intramammary infections in ewes. J. Am. Vet. Med. Assoc. 194(4):1041-1044

Hurley W.L. 1989. Symposium: Mammary Gland Function during Involution and the Declining Phase of Lactation. J. Dairy Sci. 72:16371646.

Hurley W.L. 1999. Mammary gland involution and the dry period. Lactation Biology. In: Lactation Biology Course. Departament of Animal Sciences, University of Ilinois. Disponível em <http:// classes.aces.uiuc.edu/AnSci308involution.html> acesso em 1.7.2007.

Keisler D.H., Andrews M.L. \& Mofatt R.J. 1992. Subclinical matitis in ewes and its effect on lambs performance. J. Anim. Sci. 70:16771681.

Kirk J.H., Glenn J.S. \& Maas J.P. 1996. Mastitis in a flock of milking sheep. Small Ruminant Research 22:187-191.

Krieg N.R. \& Holt J.C. 1994. Bergey's Manual of Systematic Bacteriology. 9th ed. Willians \& Wilkins, Baltimore. 1368p.

Larsgard A.G. \& Vaabenoe A. 1993. Genetic and environment causes of variation in mastitis in sheep. Small Ruminant Research 12:339347.

Lee C.S. \& Lascelles A.K. 1969. The histological changes in involution mammary glands of ewes in relation to the local allergic response. Aust. J. Exp. Biol. Med. Sci. 47:613-623.

Lee C.S. \& Outteridge P.M. 1981. Leukocyte of sheep colostrum, milk and involution secretion, with particular reference to ultrastructure and lymphocyte sub-populations. J. Dairy Res. 48:225-237.

Leitner G., Chaffer M., Zamir S., Mor T., Glickman A., Wrinkler M., Weisblit A. \& Saran A. 2001. Udder disease etiology, milk somatic cell counts and NAGase activity in Israeli Assaf sheep throughout lactation. Small Ruminant Research 39:107-112.

Lennete E.M., Balows A. \& Hansler Jr. W.J. 1985. Manual of Clinical Microbiology. 4th ed. American Society of Microbiology, Washington, D.C. 1149 p.

Mcdonald J.S. \& Anderson A.J. 1983. Intramammary inoculation of the dry cow with Staphylococcus aureus and Staphylococcus epidermidis during the nonlactating period. Am. J. Vet. Res. 44:244-246.

Mcdougall S., Murdough P., Pankey W., Delaney C., Barlow J., Murdough P.A. \& Scruton D. 2001. Relationship among somatic cell count, California Mastitis Test, impedance and bacteriological status of milk in goats and sheep in early lactation. Small Ruminant Research 40:245254.

Melchior M.B., Vaarkamp J. \& Fink-Gremmels J. 2006. Review: Biofilms, a role in recurrent mastitis infections? Vet. J. 171:398-407.

Menzies P.I. \& Ramanoon S.Z. 2001. Mastitis of sheep and goats. Vet. Clin. North Am., Food Anim. Pract. 2:333-355.

Nickerson S.C. 1987. Resistence mechanisms of the bovine udder: implications for mastitis control at the teat end. J. Am. Vet. Med. Assoc. 191:1484-1488.

Nickerson S.C. 1989. Imunological aspects of mammary gland involution. J. Dairy Sci. 72:1665-1678.

Oliver S.P. \& Sordillo L.M. 1989. Approaches to the manipulation of mammary involution. J. Dairy Sci. 72:1647-1664.

Paape M.J., Mehrzad L., Zhao X., Dettileux J. \& Burvenich C. 2002. 
Defense of the bovine mammary gland by polymorphonuclear neutrophil leukocytes. Journal of Mammary Gland Biology and Neoplasia 7(2):109-121.

Poutrel B. 1984. Udder infection of goats by coagulase-negative staphylococci. Vet. Microbiol. 9:131-137.

Prescott S.C. \& Breed R.S. 1910. The determination of the number of the body cells in milk by a direct method. J. Infect. Dis. 7:632-640.

Rejman J.J., Hurley W.L. \& Bahr J.M. 1989. Enzyme-linked immunosorbent assay of bovine lactoferin and 39-kilodalton protein found in mammary gland secretions during involution. J. Dairy Sci. 72:555-560.

Saratsis P., Leontides L., Tzora A., Alexopoulos C. \& Fthenakis G.C. 1998. Incidence risk and aetiology of mammary abnormalities in dry ewes in flocks in Southern Greece. Prev. Vet. Med. 37:173-183.

Saratsis P., Alexopoulos C., Tzora A. \& Fthenakis G.C. 1999. The effect of experimentally induced mastitis on the milk yield of dairy ewes. Small Ruminant Research 32:205-209.

Schalm O.W. \& Noorlander D.O. 1957. Experiments and observations leading to development of the California Mastitis Test. J. Am. Vet. Med. Assoc. 130(5):199-207.
Smith K.L. \& Todhunter D.A. 1982. The physiology of mammary gland during the dry period and the relantionship to infection. Proc. Annu. Meet. Natl Mastitis Council, Arlington, VA. 87p.

Tatarczuch L., Philip C. \& Lee C.S. 1997. Involution of the sheep mammary gland. J. Anat.190:405-416.

Tatarczuch L., Philip C., Bischof R. \& Lee C.S. 2000. Leukocyte phenotypes in involuting and fully involuted mammary glandular tissues and secretons of sheep. J. Anat. 196:373-379.

Tatarczuch L., Bischof R., Philip C. \& Lee C.S. 2002. Phagocytic capacity of leukocytes in sheep mammary secretions following weaning. J. Anat. 201:351-361.

Torres-Hernandez G. \& Hohenboken W. 1979. Relationships between ewe milk production and composition and pre-weaning lamb heath weight gain. J. Anim. Sci. 49:410.

Watkins G.H., Burriel A.R. \& Jones J.E.T. 1991. A field investigation of subclinical mastitis in sheep in Southern England. Brit. Vet. J. 147:413420.

Watson D.L. \& Buswell J.F. 1984. Modern aspects of sheep mastitis. Brit. Vet. J. 140:529-534. 